\title{
Tissue Modulated in vivo Raman Spectroscopy: Noninvasive Blood Analysis
}

J. Chaiken*, William F. Finney ${ }^{*}$, Douglas Hagrman*, Pamela Hagrman ${ }^{*}$, Karen Peterson*, Charles Peterson*, Yiwei Zhao , Paul E. Knudson ${ }^{* *}$ and Ruth Weinstock ${ }^{* *}$

*Department of Chemistry, Syracuse University, Syracuse, New York, 13244-4100 and LighTouch Medical, Suite 123, 600 E. Genesee Street, Syracuse, New York, 13210

**Joslin Center for Diabetes and Upstate Medical University, 750 East Adams Street, Syracuse, New York 13210

We have presented results earlier relating to the use of "tissue modulation", i.e. mechanical pressure, thermal gradients and chemical loading to differentiate tissue types for in vivo Raman spectroscopic analysis[1]. Although we expect this technique to have some general applicability, we are presently interested in obtaining Raman spectra of human capillary blood suitable for quantitative analysis of glucose and possibly other analytes.

The approach we describe uses an external cavity diode laser to excite Raman spectra containing molecule specific information relating to the probed tissues in vivo. The fluorescence that occurs simultaneously is used as a measure of the probed volume of tissue. This tissue volume is important in that it allows us to compare concentration measurements on different tissues to each other as well as concentration measurements on the same tissues at different times. The sources of the fluorescence, the factors affecting the variability of the fluorescence and the relationship between the fluorescence and the concentration of various analytes of potential interest will be discussed. In addition to the porphyrin-based emission from various sources, e.g. cytochrome, hemoglobin, we can say that amino acids and proteins and metal ions are important. Recent clinical results will be presented which suggest that this approach is quite robust and can be the basis for quantitation of in vivo Raman spectra spanning a wide range of spatial scales.

[1] J. Chaiken et al., Proc SPIE, vol. 4368, pp. 134-145,(2001) and references therein 